\title{
Chemically extracted nanocellulose from sisal fibres by a simple and industrially relevant process
}

Trifol Guzman, Jon; Sillard, Cecile; Plackett, D.; Szabo, Peter; Bras, Julien; Daugaard, Anders Egede

Published in:

Cellulose

Link to article, DOI:

$10.1007 / \mathrm{s} 10570-016-1097-5$

Publication date:

2017

Document Version

Peer reviewed version

Link back to DTU Orbit

Citation (APA):

Trifol Guzman, J., Sillard, C., Plackett, D., Szabo, P., Bras, J., \& Daugaard, A. E. (2017). Chemically extracted nanocellulose from sisal fibres by a simple and industrially relevant process. Cellulose, 24(1), 107-118. https://doi.org/10.1007/s10570-016-1097-5

\section{General rights}

Copyright and moral rights for the publications made accessible in the public portal are retained by the authors and/or other copyright owners and it is a condition of accessing publications that users recognise and abide by the legal requirements associated with these rights.

- Users may download and print one copy of any publication from the public portal for the purpose of private study or research.

- You may not further distribute the material or use it for any profit-making activity or commercial gain

- You may freely distribute the URL identifying the publication in the public portal 


\section{Chemically extracted nanocellulose from sisal fibres by a simple and \\ 2 industrially relevant process}

3

J. Trifol ${ }^{\mathrm{a}}$, C. Sillard ${ }^{\mathrm{b}}$, D. Plackett ${ }^{\mathrm{c}}$, P. Szabo ${ }^{\mathrm{a}}$, J. Bras ${ }^{\mathrm{b}}$, A. E. Daugaard ${ }^{\mathrm{a}}$

${ }^{a}$ Danish Polymer Centre, Department of Chemical and Biochemical Engineering, Technical University of Denmark, Søltofts Plads, Building 229, DK - 2800 Kgs. Lyngby, Denmark

${ }^{\mathrm{b}}$ LGP2/Grenoble INP-Pagora/CNRS, 461 rue de la papeterie, Domaine universitaire, C10065, 38402 Saint Martin d'Hères Cedex, France

${ }^{\mathrm{C}}$ Faculty of Pharmaceutical Sciences, University of British Columbia, 2405 Wesbrook Mall, Vancouver, BC V6T 1Z3, Canada

\section{Keywords:}

Sisal fibres; Cellulose nanofibres (CNFs); Cellulose films; acetylation, nanofibers

\section{Abstract:}

A novel type of acetylated cellulose nanofibre (CNF) was extracted successfully from sisal fibres using chemical methods. Initially, a strong alkali treatment was used to swell the fibres, followed by a bleaching step to remove the residual lignin and finally an acetylation step to reduce the impact of the intermolecular hydrogen bonds in the nanocellulose. The result of this sequence of up-scalable chemical treatments was a pulp consisting mainly of micro-sized fibres, which allowed simpler handling through filtration and purification steps and permitted the isolation of an intermediate product with a high solid content. An aqueous dispersion of CNF could be obtained directly from this intermediate pulp by simple magnetic stirring. As a proof of concept, the dispersion was used directly for preparing a highly translucent CNF film, illustrating that there is no large aggregates in the prepared CNF dispersion. Finally, CNF films with alkali extracts were also prepared, resulting in flatter films with an increased mass yield and improved mechanical strength.

\section{INTRODUCTION}

Cellulose is the most abundant bio-derived polymer in the world, with a yearly production of about $10^{11}$ tons (Azizi Samir et al. 2005). This production originates mainly from plants, but there are other sources of cellulose such as bacteria, tunicates and algae(Moon et al. 2011). Cellulose has the empirical formula $\left(\mathrm{C}_{6} \mathrm{H}_{10} \mathrm{O}_{5}\right)$ and is a linear homopolysaccharide with hundreds to thousands of glucose units connected through 1-4- $\beta$-glucosidic bonds. Cellulose is semicrystalline and therefore contains both amorphous and crystalline domains of various types depending on the source of cellulose. Due to the recently increased focus on sustainability, lignocellulosic materials in general, and cellulose in particular, have been investigated widely in search of novel application fields such as biofuels(Baker and Keisler 2011), polymer reinforcement(Saheb and Jog 1999) and biomedical applications(Czaja et al. 2007) (Lin and Dufresne 2014). A turning point in this development occurred when Herrick et al. (Herrick et al. 1983) successfully isolated microfibrilated cellulose using mechanical methods to break up the hierarchical structure of cellulose. The cellulosic fibres can be considered bundles of nanosized 
cellulosic fibres (CNFs) comprising of cellulosic fibrils with a high aspect ratio, having diameters on the nanoscale and lengths on the microscale. CNFs have been shown to have very interesting properties, such as a specific Young's modulus that is 3.4 times higher than that of steel(Eichhorn et al. 2009). Research into applications of the nanosized cellulosic materials has increasingly caught the interest of the scientific community(Lavoine et al. 2012), and the subject has been widely studied for applications such as hydrogels(Chang and Zhang 2011), aerogels(Fischer et al. 2006), barrier coatings(Minelli et al. 2010) and polymer reinforcement(Siró and Plackett 2010). Films containing such nanofibres are reported to have very good mechanical properties(Siró and Plackett 2010), high transparency(Siró et al. 2011), good oxygen barrier properties at low relative humidity as well as medium water vapour barrier properties(Lavoine et al. 2012) due to the high water uptake of the nanofibres(Minelli et al. 2010).

In order to bring CNF applications to market, an industrially relevant method that can extract and break up the strong association between fibres to prepare them for use in composites is required. Usually, the procedures for obtaining CNFs are based on applying high shear forces to extracted fibres e.g. by grinding, micro fluidisation, homogenisation or other similar techniques. These methods are usually highly energy demanding and are performed in high dilution or using processes that are not directly up-scalable or cost-effective. Therefore extensive research has been invested in finding methods for pre-treatment, such as enzymatic treatments(Henriksson et al. 2007) or using (2,2,6,6-tetramethylpiperidin-1-yl)oxyl (TEMPO)(Saito et al. 2006) for oxidation of the cellulose to decrease the energy consumption and weaken the hydrogen bonds between fibres(Qing et al. 2013; Abdul Khalil et al. 2014). Here a method to obtain partially acetylated CNF, by employing a simple chemical treatment followed by a low energy dispersion step, is presented. The intermediary pulp achieved after the chemical treatments is easy to filter and dry, which makes it highly suitable for transportation.

\section{EXPERIMENTAL}

\subsection{Materials and methods}

Cellulose nanofibres were extracted from sisal, which was kindly supplied by Expor Sisal S.L., while sodium hydroxide, nitric acid (ACS reagent, 70\%) and acetic acid (99\%-100\%) were purchased from Sigma Aldrich and sodium chlorite ( $25 \mathrm{wt} \%$ in water) was obtained from Merck. All of the reagents were used as received. If not specified, the analysis of CNF were done after centrifugation.

\subsubsection{Extraction and isolation of the acetylated CNF pulp (SMBA)} Sisal fibres ( $50 \mathrm{~g}$ ) were cut and rinsed with an aqueous solution of sodium hydroxide $(1.5 \mathrm{~L}, 2 \mathrm{wt} \%)$ at $23^{\circ} \mathrm{C}$ for 16 hours. The rinsed fibres were isolated by filtration and washed with distilled water until constant $\mathrm{pH}$ of the washing water was achieved. The alkali treatment (mercerisation) was repeated three times (1.5 hours at boiling temperature) with a stronger alkali solution (1.5 L, $10 \mathrm{wt} \%$ ) followed by filtration, after which the pulp (SM) was suspended in distilled water $(1.25 \mathrm{~L})$, and the temperature was increased to $70^{\circ} \mathrm{C}$. Once this temperature was reached, acetic acid $(8 \mathrm{~mL})$ followed by sodium chlorite $\left(\mathrm{NaClO}_{2}, 25 \mathrm{wt} \%, 40 \mathrm{~mL}\right)$ was added once every hour for 7 hours. Finally, the wet bleached pulp (approximately $30 \mathrm{~g}$ on dry mass) was isolated by filtration and washed with distilled water until a constant $\mathrm{pH}$ was reached (SMB). Thereafter, this pulp was suspended in a mixture of nitric acid $(150 \mathrm{~mL})$ and acetic acid $(900 \mathrm{~mL})$, and the mixture was stirred at boiling temperature for 90 minutes. The mixture was cooled by dilution with cold distilled water (ratio 1:5) and the acetylated pulp was 

acetylated pulp (SMBA) was used without further purification.
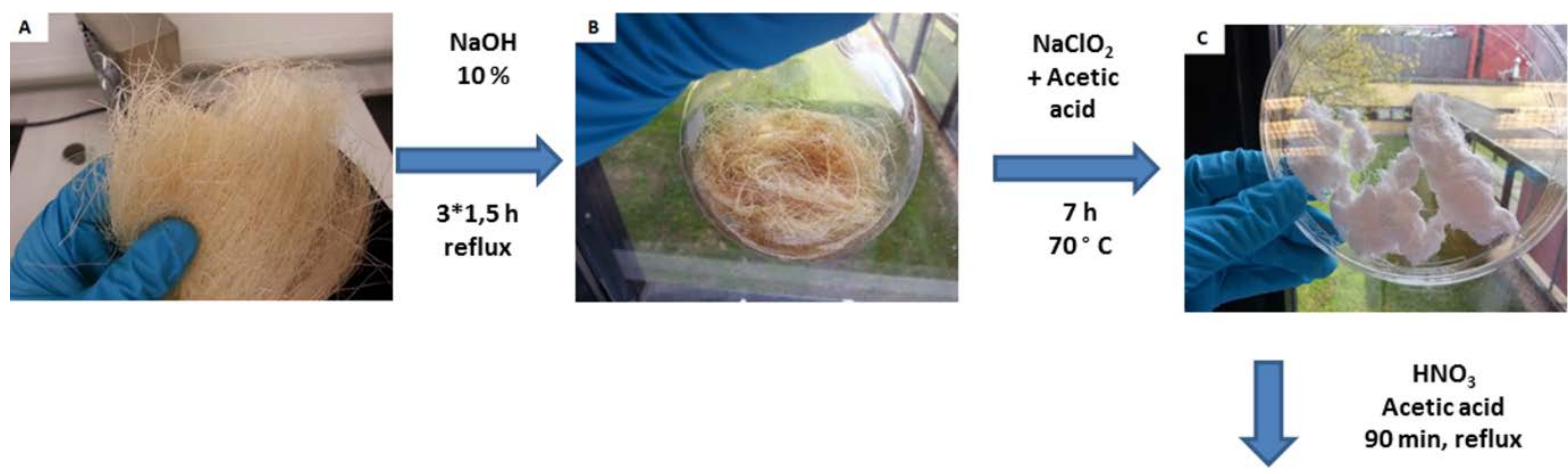

$\mathrm{HNO}_{3}$ Acetic acid $90 \mathrm{~min}$, reflux
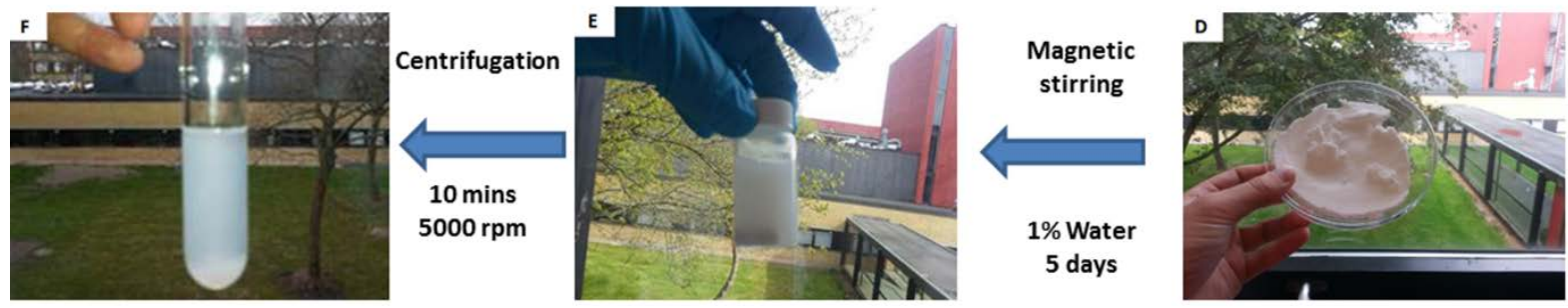

Figure 1. Overview of the CNF extraction protocol. A) Sisal fibres (S); B) Sisal fibres after alkali treatments (SM); C) Sisal fibres after mercerisation and bleaching (SMB); D) Sisal fibres after mercerisation, bleaching and acetylation (SMBA); E) SMBA after dispersion in water (CNF) and F) Stable CNF dispersion after centrifugation.

2.1.2 Extraction of lignin and hemicellulose for compounding (residue solution)

Sisal fibres (35 g) were mixed with an aqueous solution of sodium hydroxide ( $300 \mathrm{~mL}, 10 \mathrm{wt} \%$ ), and the mixture was refluxed for 2 hours. The fibres were filtered off and the filtrate was cooled and dialysed in a regenerated cellulose membrane (Cellu Sep T3, MWCO 12,000-14,000) against distilled water until pH was constant. The dialysed residue containing cellulose, lignin and hemicellulose was used without further purification.

\subsubsection{Preparation of CNF films}

An aqueous dispersion of CNF in water (1 wt\%) was prepared by dilution of the SMBA pulp with distilled water followed by magnetic stirring for five days in an Erlenmeyer flask. The dispersion was centrifuged at $5000 \mathrm{rpm}$ for 10 minutes, and the supernatant ( $0.8 \mathrm{wt} \%$ ) was transferred to a Teflon mould. A translucent CNF film was formed by concentration of the solution in a climatic chamber for one week at $19^{\circ} \mathrm{C}$ and $65 \%$ relative humidity $(\mathrm{RH})$, resulting in a translucent film with a thickness of 20-30 $\mu \mathrm{m}$.

\subsubsection{Preparation of the CNF/residue films}

$\mathrm{CNF} /$ residue films were prepared from the aqueous CNF dispersion as described above for the CNF films. from the mercerisation) to obtain a mixture with $70 \mathrm{wt} \% \mathrm{CNF}$ and $30 \mathrm{wt} \%$ residue. The mixture was cast in a 
Teflon mould and dried in a climatic chamber for one week at $19^{\circ} \mathrm{C}$ and $65 \% \mathrm{RH}$, resulting in the formation of a translucent CNF/residue film with a thickness of 20-30 $\mu \mathrm{m}$.

\subsection{Characterization}

Thermogravimetric analysis (TGA) was performed on a thermal TGA Q500 (TA) instrument from $25-600^{\circ} \mathrm{C}$ with a heating rate of $10 \mathrm{~K} / \mathrm{min}$ under nitrogen flow. Fourier transform infrared spectroscopy (FT-IR) was obtained by triplicate on a Thermo-Fisher is50 FT-IR spectrometer equipped with a universal attenuated total reflection (ATR) sampling accessory with a diamond crystal at a resolution of $4 \mathrm{~cm}^{-1}$ in the range of 500$4000 \mathrm{~cm}^{-1}$

The rheological data was obtained with a TA Instruments AR2000 controlled stress rotational rheometer using a cone-and-plate geometry. The aluminium cone had a diameter of $60 \mathrm{~mm}$ and a $1^{\circ}$ cone angle. Viscosity measurements were obtained in steady shear at $25^{\circ} \mathrm{C}$.

Scanning electron microscopy (SEM) of the sisal and processed fibres was carried out using a Hitachi T3030 with a $5 \mathrm{kV}$ field. The CNF was characterised by SEM on an FEI Quanta 200 ESEM FEG. Transmission electron micrographs were obtained using a Hitachi HT770 microscope operating at $100 \mathrm{kV}$. A drop of an aqueous CNF suspension (0.2 \% CNF) was deposited on a 200 mesh carbon/formvar copper grid (TED PELLA, USA) and imaged without the addition of staining agents or other chemicals.

X-ray diffraction was performed using a Panalytical X'Pert Pro MPD-Ray diffractometer with an Ni-filtered Cu K $\alpha$ radiation ( $\lambda=1.54 \AA$ ) source, a voltage of $45 \mathrm{kV}$, a current of $40 \mathrm{~mA}$ and scans from $5^{\circ}$ to $60^{\circ}$. The crystallinity index of the extracted fibres was calculated using the Buschle-Diller and Zeronian equation.

$$
I_{\mathrm{C}}=1-\frac{I_{1}}{I_{2}}
$$

Where $I_{1}$ is the peak at $2 \theta=18.8^{\circ}$ (amorphous peak) and $I_{2}$ is the peak at $2 \theta=22.8^{\circ}$ (crystalline peak), $I_{c}$, the crystalline index.

Optical properties were measured in triplicates using a UV-Vis spectrometer (Polar Star Omega) in the range of $200 \mathrm{~nm}-1000 \mathrm{~nm}$ and a Gardener Haze-Gard Plus to analyse the transmittance, haze and clarity of the specimens. Transmittance is the percentage of light transmitted through the sample, haze the amount of transmitted light that is scattered more than $2.5^{\circ}$ and clarity the amount of transmitted light that is scattered less than $2.5^{\circ}$.

The surface properties of CNF films were estimated by advancing contact angle (CA) measurements on a Dataphysics Contact Angle System OCA20. Specimens of 2 x $1 \mathrm{~cm}$ were placed on a glass slide and a drop of water $(6 \mu \mathrm{L})$ was deposited on the specimen surface. Thereafter, the needle was placed into the drop and the advancing CA was determined as the constant value obtained with a flow of $0.5 \mu \mathrm{L} / \mathrm{s}$. The CA was determined as an average of at least five measurements. 
131 Water absorption analysis was performed in duplicates based on the mass increase of samples (100-300 mg)

132 conditioned in a climatic chamber overnight at $23^{\circ} \mathrm{C}$ with a relative humidity of $10 \%, 25 \%, 50 \%$ and $75 \%$,

133 respectively.

134 The mechanical properties were measured by duplicate in a DMA RSA3 (TA Instruments, USA) working in 135 tensile mode. The specimens (2 cm length and $0.5 \mathrm{~cm}$ width) were preconditioned at $23{ }^{\circ} \mathrm{C}$ and $50 \% \mathrm{RH}$ for 24 136 hours prior to the measurement, which was carried at a speed of $1 \mathrm{~mm} / \mathrm{min}$ with a distance between the fixtures 137 of $10 \mathrm{~mm}$.

\section{RESULTS AND DISCUSSION}

\subsection{From raw materials to nanomaterials}

141 The developed method employs simple and industrially relevant processes for the conversion of sisal fibres into

142 CNF. Each of the steps is described in detail in the experimental section, as shown in Figure 1. An initial alkali 143 treatment was used to remove oil residues and impurities from the fibres. Three subsequent stronger alkali 144 treatments of sisal (S), to acquire mercerised fibres (SM, B in Figure 1), was used to swell the fibres and to 145 extract lignin and hemicellulose from the fibres. The repeated mercerisation minimized adsorption of these 146 impurities on the surface of the fibres compared to one longer mercerization step. After alkali treatment the 147 fibres were light-brown in colour, which is attributed to deposition of the extracted lignin on the surface of the 148 fibres. The deposited lignin was removed in the following bleaching step (SMB, C in Figure 1), and finally the 149 influence of hydrogen bonds between the fibres was reduced through acetylation of the fibres (SMBA, D in 150 Figure 1). This extraction protocol is based on two separate modifications of the fibres. Firstly, the alkali 151 treatment swells the fibres, breaks the strong association, due to hydrogen bonds, between the cellulose chains 152 and opens up the structure to additional chemical treatments (Mwaikambo and Ansell 1999). Secondly, in the 153 swollen state, the hydroxyl groups from the cellulose nanofibres are grafted with acetate groups, which thereby 154 permanently reduces the energy required to break the strong association between cellulose nanofibres in 155 subsequent processing steps.

156 The result of this sequence of chemical treatments was a pulp consisting mainly of modified micro-sized fibres, which is easy to transport or store as a precursor for later preparation of CNF dispersions. An aqueous dispersion of CNF was obtained directly from this intermediate by magnetic stirring (product E in Figure 1), and any agglomerates were removed by centrifugation. The yield of cellulosic material after each step of the extraction protocol is shown in Figure 2. 


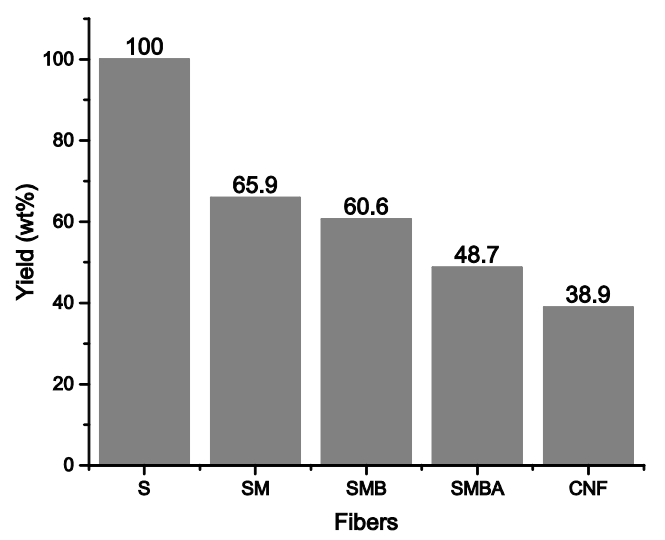

161

Figure 2. Mass yield of the treated fibres after drying of a sample of the respective suspensions, where the CNF is the supernatant obtained after centrifugation in the last step (all results are on a dry basis).

164 The amount of extracted cellulose fibres depends strongly on the type of fibres used for the process. Sisal fibres are generally reported to consist of about 60-70 wt\% cellulose, 10-15 wt\% hemicellulose and 8-12 wt\%

166 lignin(Bismarck et al. 2001; Mondragon et al. 2014). After all of the chemical treatments and purification steps

167 that removes the majority of the lignin and hemicellulose, $39 \mathrm{wt} \%$ of the original sisal fibres were converted into

168 a stable CNF dispersion, which corresponds to an extraction of approximately $60 \mathrm{wt} \%$ of the total amount of

169 cellulose from the sisal fibres. This compares to other extraction protocols, where e.g. $65 \mathrm{wt} \%$ of cellulose

170 nanofibres have been extracted from cotton(de Morais Teixeira et al. 2010) or 50-60 wt\% were extracted from

171 softwood(Tejado et al. 2012).

$172 \quad 3.2$ Characterization of the pulp

173 The isolated material was characterised by SEM after each step, in order to illustrate the effects of each of the 174 treatments, as shown in Figure 3. 


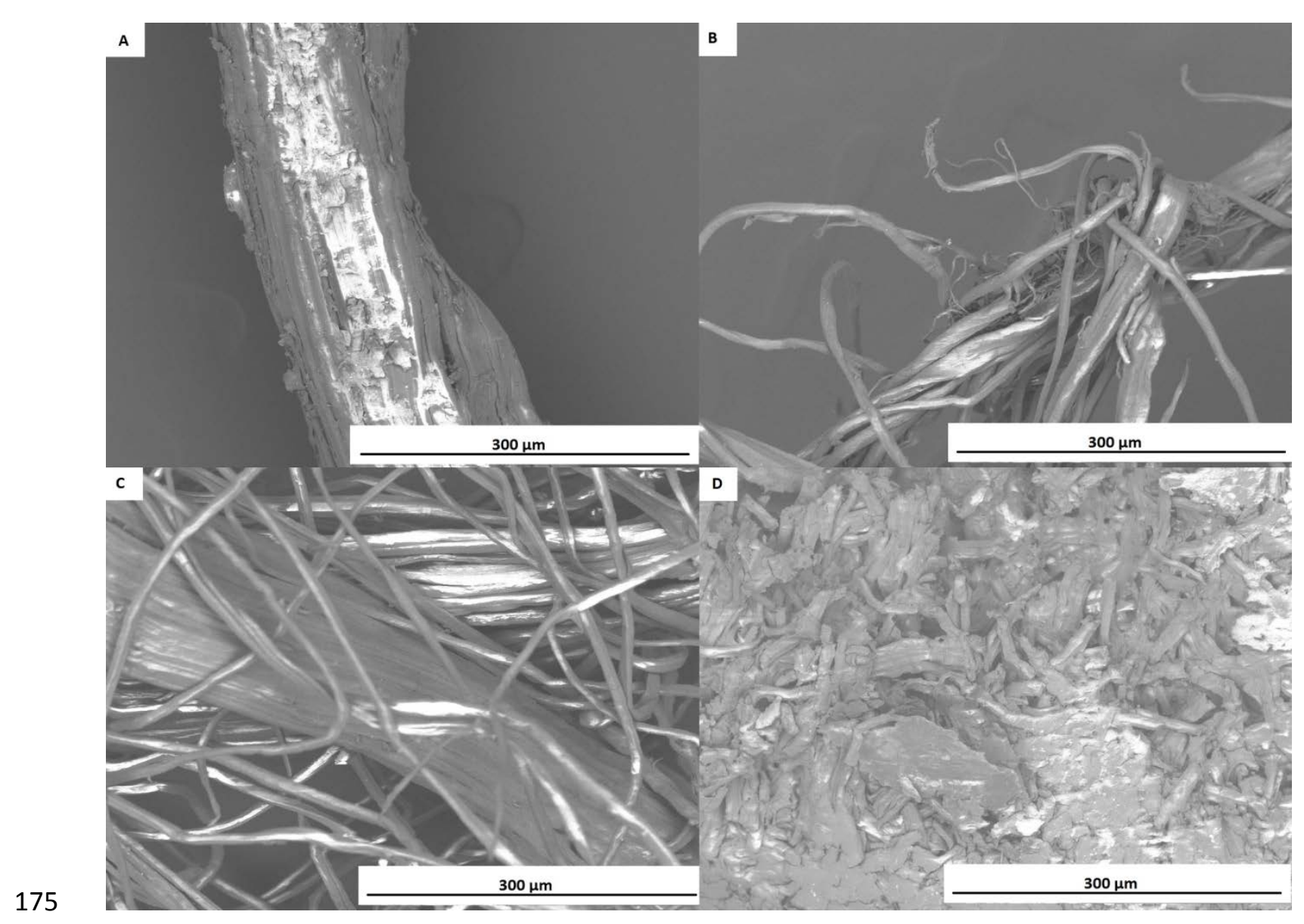

Figure 3. SEM pictures of the fibres at the various stages of the process showing the transgression from large fibre bundles to the fully treated acetylated fibres in the final pulp. A) Sisal fibres B) fibres after alkali treatments C) fibres after bleaching D) fibres after acetylation.

179 The micrographs in Figure 3 show how each step in the process affects the fibres. The strong alkali treatment swells the fibres and results in the formation of free individual fibres (SM). In the bleaching step even more separated fibres are produced (SMB) due to the removal of the remaining lignin, which reduces the cohesion between the fibrils. Finally after the acetylation step the structure of the macroscopic sisal fibres have been completely removed, resulting in the formation of a more uniform mass consisting of much smaller fibres. The results of the extraction protocol in terms of chemical and thermal properties can be seen in Figure 4.
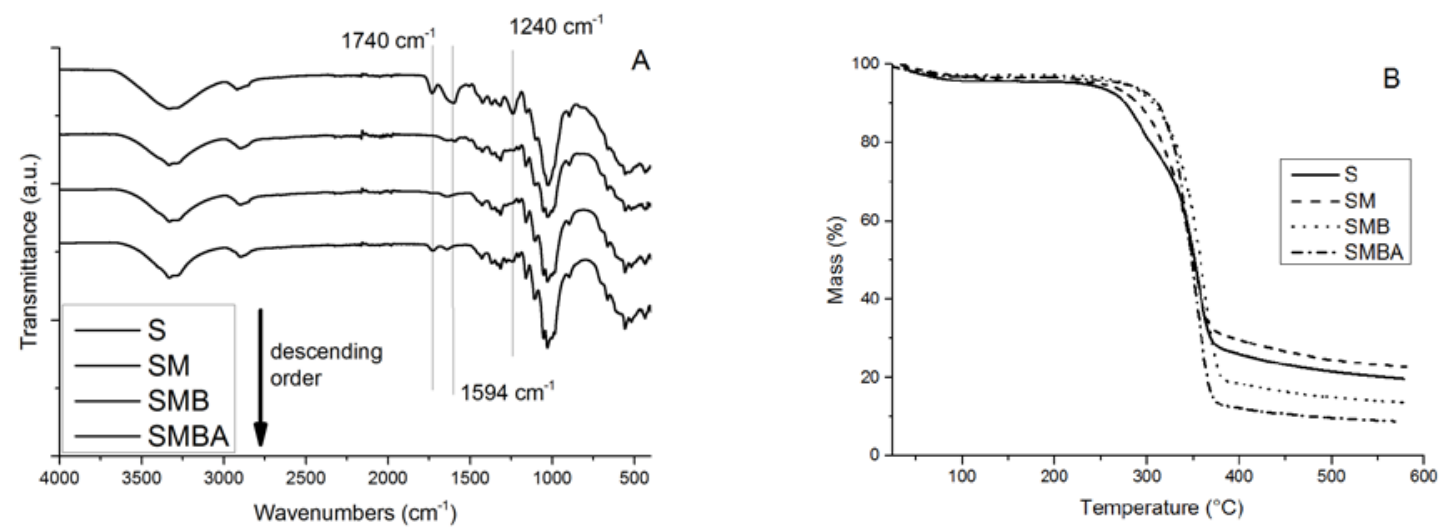

Figure 4. Analysis of the extracted CNF at each step in the process by FT-IR (A) and by TGA (B). 
In Figure 4a the chemical changes to the fibres are illustrated by changes in the IR spectra. Here the removal of the majority of hemicellulose during the first alkali treatment from S to SM can be seen through disappearance of the peaks at $1740 \mathrm{~cm}^{-1}$ (which are related to acetyl and ester groups, characteristic of hemicellulose) and at $1240 \mathrm{~cm}^{-1}$ (C-O stretching vibration of the hemicellulose). In addition to this, the disappearance of the peak at $1594 \mathrm{~cm}^{-1}$ (related to the C-C in the plane symmetrical stretching of aromatic rings, characteristic of lignin) from SM to SMB shows that the most part lignin has been removed from the fibre. This is corroborated by the fact that the fibres are completely white after bleaching. The removal of a large part of the hemicellulose is confirmed by TGA in Figure 4b. Hemicellulose usually degrades between $200-400^{\circ} \mathrm{C}$, which results in an increase in the onset of thermal degradation after both the alkali treatment and the bleaching step compared to the pure sisal fibres. Finally, acetylation of the pulp in the last step of the process is confirmed by reappearance of the peak at $1740 \mathrm{~cm}^{-1}$, attributed to new acetate groups. The intensity of the carbonyl stretch indicates that only a partial acetylation has taken place, which was also confirmed by the low degree of substitution (10\%) determined using the method published by Kim et al. (Kim et al. 2002). The extracted residues were also analysed (SI-Figure 1) and it was found that the alkali treatments removed not only the majority of lignin and hemicellulose, but also significant amounts of cellulose.

\subsection{Characterization of the CNF}

Introduction of acetate groups on the surface of the fibres, however, is not enough to fully separate the nanofibres. This acetylation results in reduced cohesion between the nanofibres, but a very small amount of energy is still required in order to separate the fibres. Magnetic stirring is sufficient to separate the nanofibres as shown by the increase in viscosity of a 4 wt\% SMBA dispersion stirred for respectively 2 and 20 hours, as shown in Figure 5.

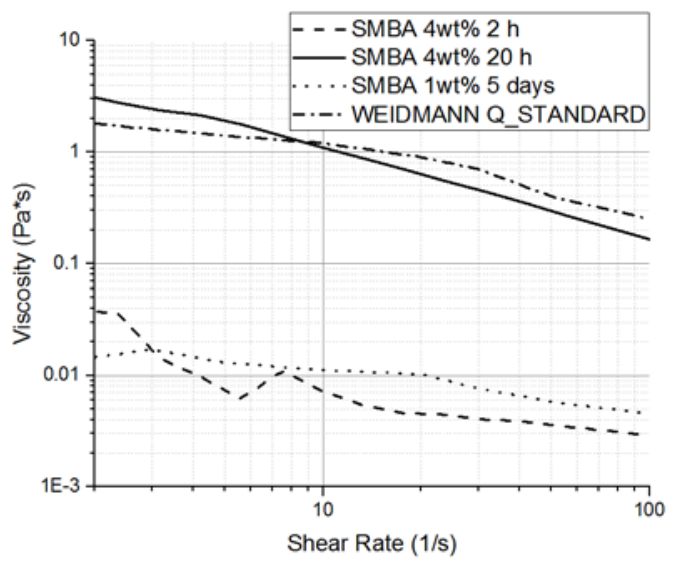

Figure 5. Viscosity of a $4 \mathrm{wt} \%$ SMBA solution in water after magnetic stirring for 2 hours and 20 hours compared to a commercial CNF (Weidmann Q standard) and to a 1wt\% CNF solution that has been stirred for 5 days.

210 The viscosity is dramatically increased between 2 and 20 hours of magnetic stirring, due to dispersion of the

211 CNF in water. With continued stirring the fibres becomes gradually more and more separated and eventually the sample reaches the rheological percolation threshold, resulting in formation of a CNF network and a significant increase in viscosity. The viscosity of the resulting dispersion after 20 hours was similar to a commercially 
available CNF produced by Weidmann (Q standard). The increased viscosity proves that the very soft mechanical treatment successfully disperses the CNF. However, at $4 \mathrm{wt} \% \mathrm{CNF}$ the viscosity was so high after 20 hours that magnetic stirring was no longer powerful enough to efficiently stir the suspension (it formed a hydrogel), which prevents the complete dispersion of the CNF. Therefore the concentration was reduced to $1 \mathrm{wt} \%$, which it was possible to stir for 5 days without reaching the rheological percolation threshold. Samples of this solution were analysed by optical microscopy after different stirring times as shown in Figure 6.

220
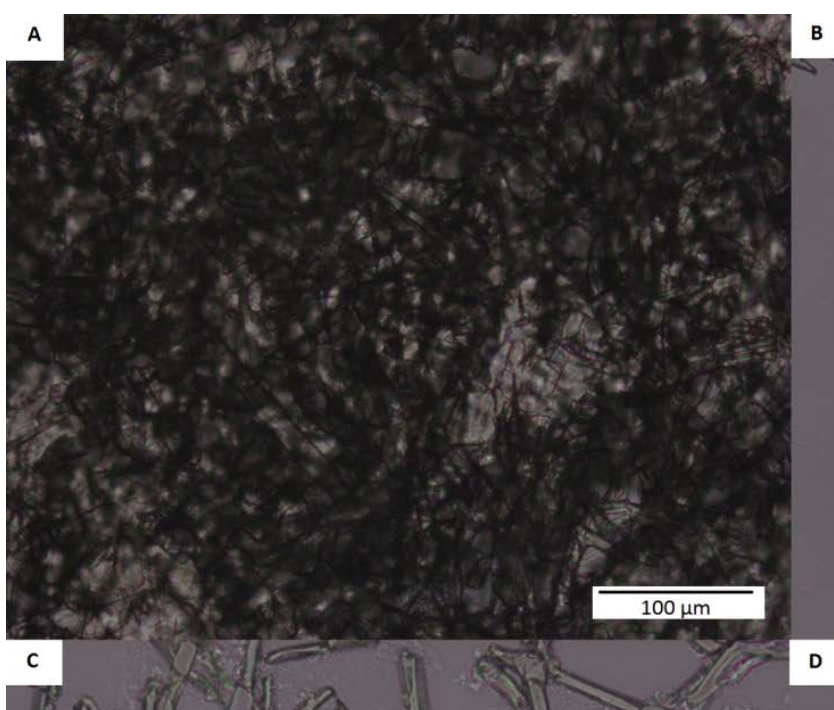

B

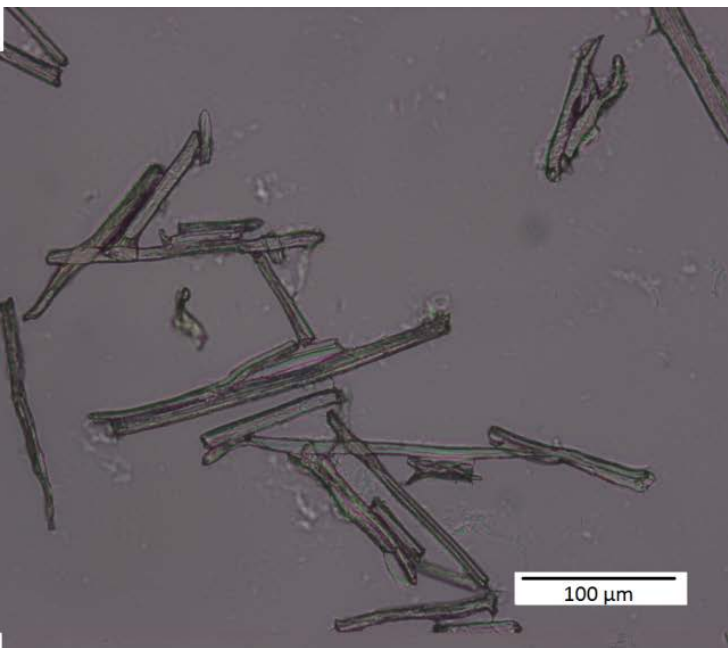

D

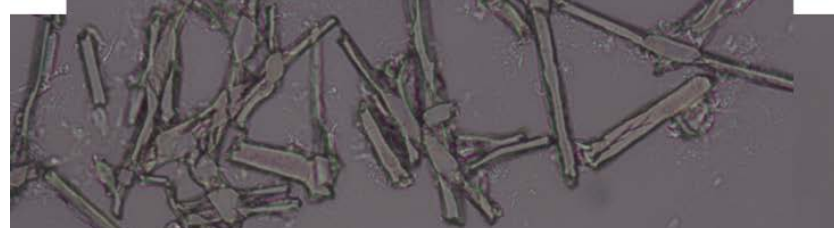

Figure 6. Optical micrographs of a $1 w t \%$ mixture of the acetylated pulp (SMBA) after magnetic stirring in water for 0 min (A), 15 min (B), 1 hour (C) and 48 hours (D).

224 Simple magnetic stirring breaks up the aggregates and ultimately results in a stable aqueous dispersion of the nanofibres after 48 hours. After two days of magnetic stirring, it is no longer possible to see large fibres in the optical microscope, thus suggesting that the majority of the fibres are below microscale in size.

227 In order to evaluate the dimensions of the prepared nanofibres, a drop of a $0.6 \mathrm{wt} \%$ CNF dispersion was casted on an aluminium film, resulting in the formation of a thin film with a film thickness of around $100 \mathrm{~nm}$. The prepared film was sputtered and investigated by SEM, which showed a uniform distribution of nanofibres on the 

electron microscopy (TEM) as shown in Figure 7.

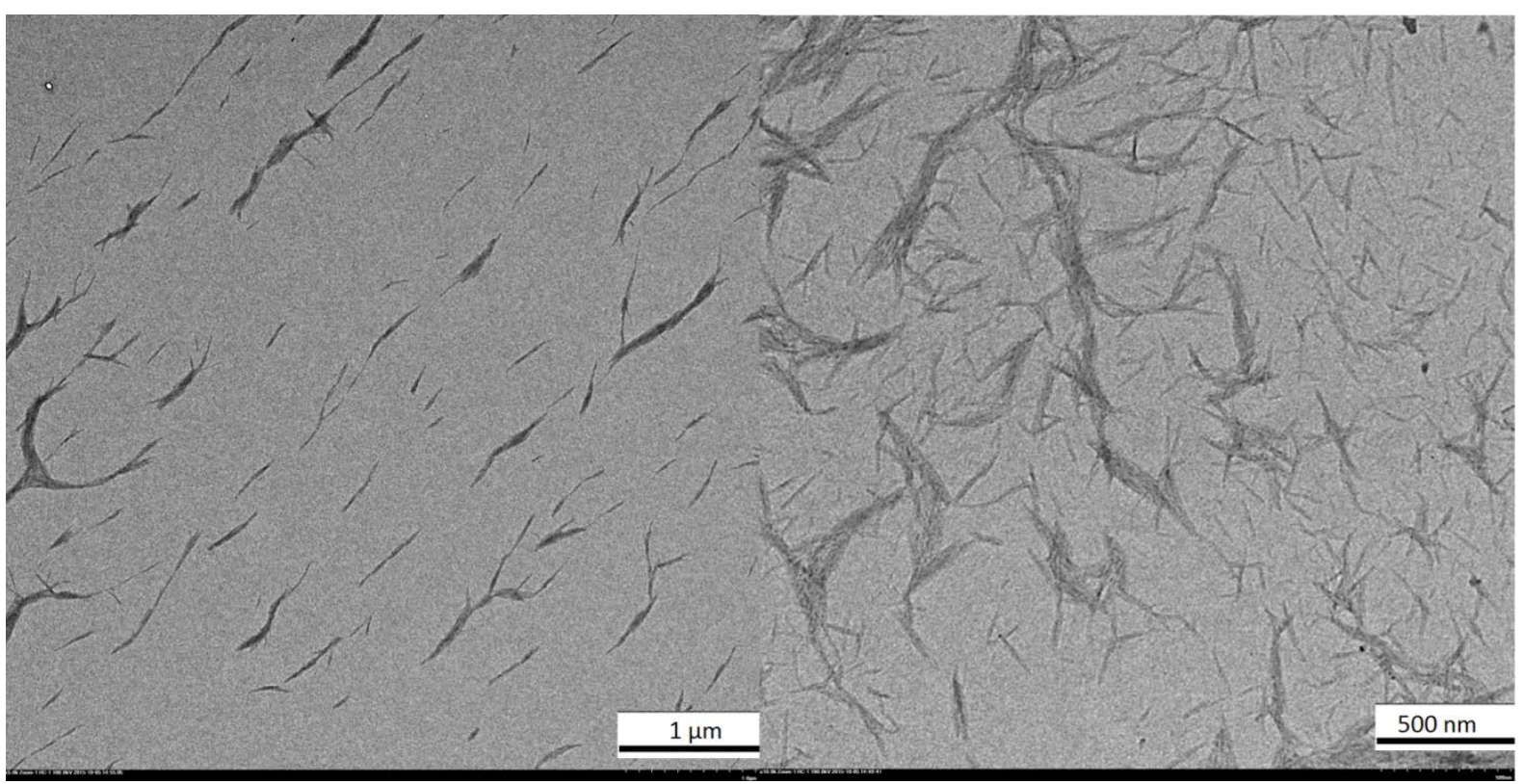

Figure 7. Analysis of the structure of the isolated CNF by TEM at different magnifications.

From both SEM and TEM it is clear that no large fibres are present in the film casted from the dispersion. The fibres are estimated to have a diameter of $27+/-13 \mathrm{~nm}$ and a length of $658+/-290 \mathrm{~nm}$ with minor aggregates of approximately $160+/-75 \mathrm{~nm}$ in diameter and $0.90+/-0.42 \mu \mathrm{m}$ in length. The fibres are shorter than what has been obtained when CNFs are prepared by using, for example, TEMPO-mediated oxidation and mechanical methods such as homogenisation, ultrasound or grinding (Moon et al. 2011), which is attributed to the harsh conditions employed during the extraction process.

The processing of cellulosic materials is known to affect the crystallinity, and it was therefore investigated by $\mathrm{X}$-ray diffraction (XRD), as shown in Figure 8.

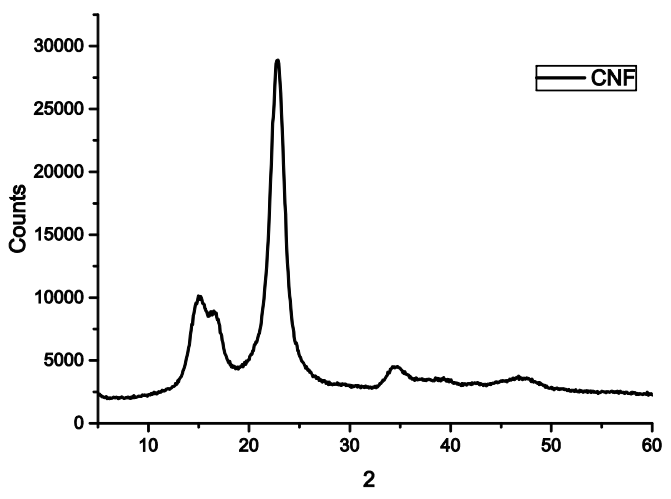

Figure 8. XRD analysis of the purified CNF.

244 The XRD spectrum shows the expected peaks from a cellulose material with peaks at $2 \theta=15.13$ and $2 \theta=22.88^{\circ}$.

245 The degree of crystallinity of the extracted fibres was calculated based on the peak at $22.88^{\circ}$, and it was determined to be $84.2 \%$. This value is comparable to the crystallinities reported in the literature where sisal- 
247 based CNF was reported to have a crystallinity of 93\% (Siqueira et al. 2010) and from TEMPO oxidized CNF

248 having a crystallinity of 59-92\% (Lavoine et al. 2012).

\section{$249 \quad 3.3$ CNF film properties}

250 The CNF dispersion was used to prepare large CNF films by solution casting of either the prepared CNF

251 dispersion directly or by combination of the CNF dispersion and the extracted residues. The prepared films have

252 a high clarity and good transparency as shown in Figure 9.
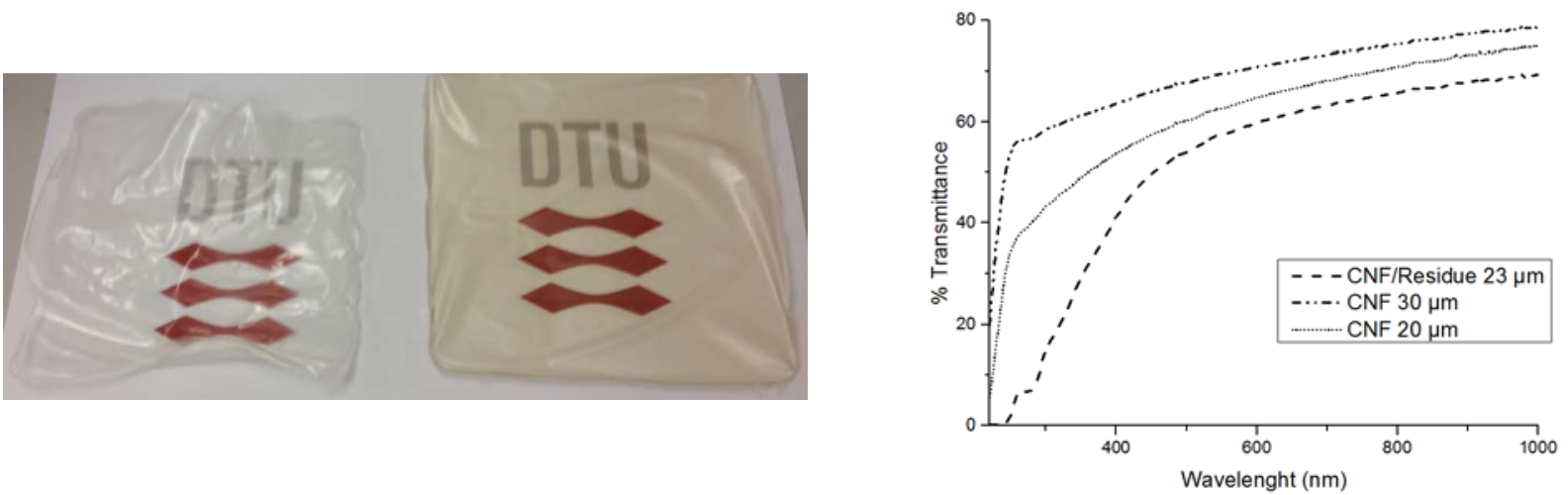

Figure 9. Left: Optical image of the prepared film (pure CNF on the left and CNF with residue solution on the right); Right: Transmittance of the CNF films determined by UV-vis spectroscopy.

255 The film prepared from the pure CNF is fully translucent, and has a high clarity (69,8\% clarity, 26,7\% haze)

256 whereas the film with the added residues (extracted cellulose, lignin and hemicellulose from the mercerisation

257 step) resulted in a light-brown but more uniform film with a slightly reduced clarity (44,6\% clarity, 36,2\% haze)

258 (see Table 1, supporting information). The CNF/Residue film additionally had a decreased UV transmittance,

259 due to the presence of lignin that acts as a UV absorber and as antioxidant. Ultimately, the CNF are intended for

260 use in composites for packaging materials and for this application a reduced UV transmittance as well as the

261 antioxidative properties of the lignin are interesting properties. The residues were also incorporated into the

262 film, in order to investigate if they could potentially decrease the water sorption of the films as well as to

263 investigate any effects on film forming. The cross-sectional view of the films by SEM can be seen in Figure 10. 

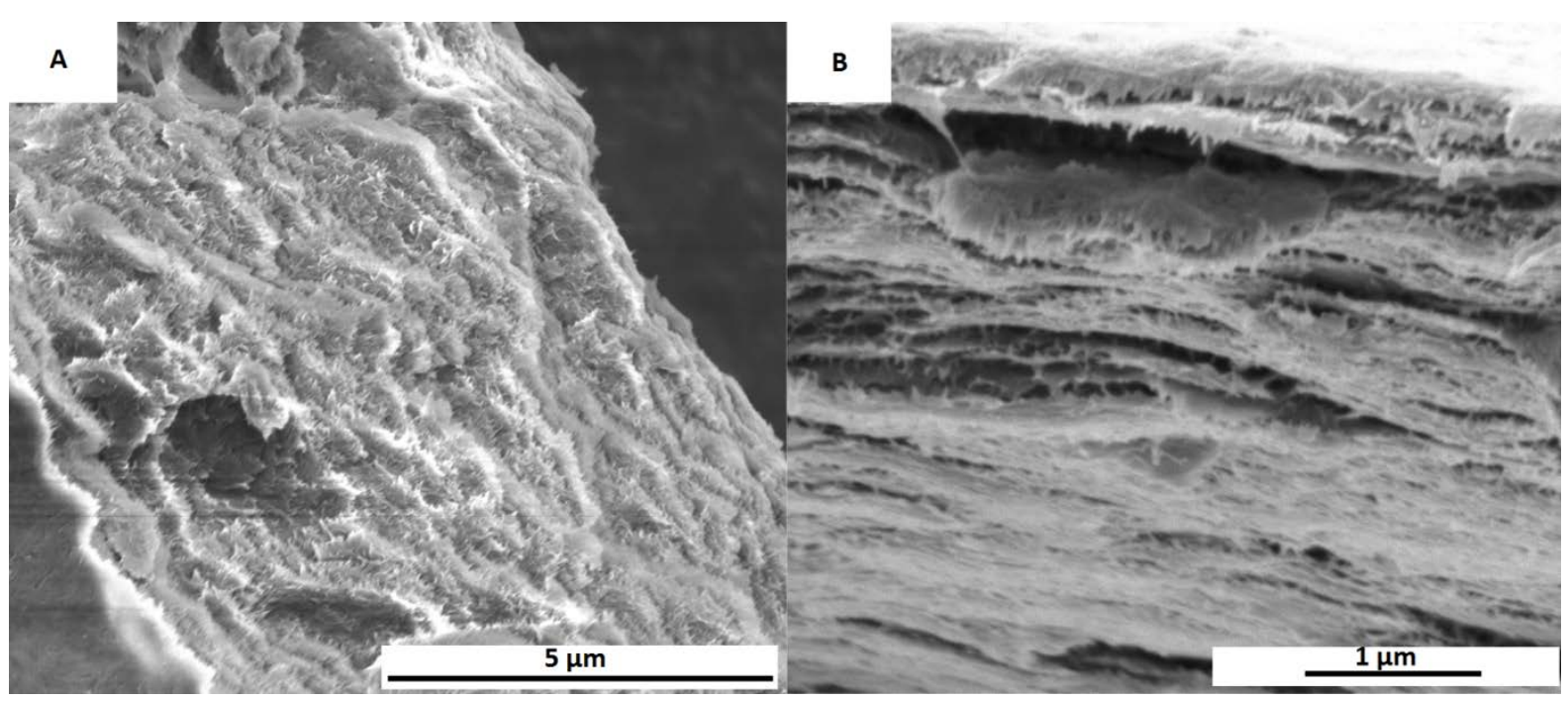

Figure 10. SEM analysis of a fractured cross section of a) Neat CNF film and B) CNF/Residue film.

The SEM micrographs show that there are no large agglomerates on the fractured interphase from the films, (SEMs of the surface view of the films can be seen in the supporting information in SI-Figure 3 and 4). The micrographs also show a clear differences in the layered structures of the two films, where the CNF/Residue film is clearly much more compact compared to the pristine CNF film, which appears to have a more open structure. This feature of the CNF/Residue film is attributed to the lignin and hemicellulose in the residue that work as binders and make a stronger bonding with CNF through hydrogen bonding, which results in a reduced swelling of the CNF during the drying stage.

The mechanical properties of the films were investigated (SI-Table2), showing that in particular the pure CNF film had lower tensile strength and elongation at break compared to other nanocellulose films(Henriksson et al. 2008; Siró et al. 2011). This is attributed to the partial acetylation of the fibres, which results in less hydrogen bonding between the fibres in the neat film. However, the degree of substitution is not high enough to affect the transparency of the film, as would be expected from a fully acetylated CNF. The pure CNF films were very brittle and had an uneven surface, which made the mechanical analysis very difficult. In contrary to this, the $\mathrm{CNF} /$ Residue films were more uniform and easier to handle, which resulted in a significant increase in tensile strength and elongation. CNF films, apart from their brittleness, have been reported to have good properties at low relative humidity, but their barrier properties dramatically decreases with increasing relative humidity(Aulin et al. 2010; Minelli et al. 2010). For this reason, any process that could decrease water sorption would be very useful for new applications of CNF films. The CNF nanomaterials prepared here are partially acetylated, which could potentially affect water sorption. In an effort to elucidate this, the advancing water contact angles of the prepared films were determined. Both films have comparable water contact angles of $48.8^{\circ}+/-2.7$ for the CNF film and $52.4^{\circ}+/-1.1$ for the CNF/Res film, which is similar to other non-functionalised nanocelluloses $\left(41.2^{\circ}\right.$ (Rodionova et al. 2010), 50-60(Siqueira et al. 2010), 50(Wu et al. 2014)). Apparently the partial acetylation does not significantly increase the hydrophobicity of the thin films, which corroborates the low degree of substitution (10\%). The actual water sorption of the films can be seen in the supporting information (SI-Figure 5). The observed water sorption, approximately $2,6 \%$ at $23^{\circ} \mathrm{C}$ and $50 \%$ rel. humidity for both types of films, was slightly decreased compared to other nanocellulose films, where CNF and nanocrystalline cellulose have been 
shown to have water sorption of $6.5 \%$ at $25^{\circ} \mathrm{C}$ and $50 \%$ rel. humidity (Belbekhouche et al. 2011), or $4 \%$ at $35^{\circ} \mathrm{C}$ and 50\% rel. humidity for enzymatically pre-treated CNF(Minelli et al. 2010).

The mechanical properties of these materials are too poor to allow a direct application of these materials. However, the process produces partially acetylated CNFs, which makes these amphiphilic materials easier to disperse in organic media, and thereby makes them well suited for use as reinforcement agents in e.g. poly(lactic acid) (PLA). Recently CNF composites in PLA were shown to have superior thermomechanical resistance and enhanced barrier properties (PLA/CNF 1\% showed a 64\% of decrease on oxygen transmission rate, a 46\% of decrease on water vapour transmission rate)(Trifol et al. 2016a), which were greatly improved when both CNF and a commercially available clay (C30B) were used to reinforce PLA (PLA/CNF 5\%/C30B 5\% showed a a reduction of up to $90 \%$ in OTR and a further reduction in the water vapour transmission rate (WVTR) of up to 76\% (Trifol et al. 2016b).

\section{CONCLUSIONS}

In this study a method is presented whereby a partially acetylated CNF can be prepared by employing a chemical treatment protocol. This method of extracting cellulose nanofibres has several advantages. The raw pulp produced after the chemical treatments is produced in a high yield of $48 \%$ and can be easily filtered and purified. The isolated SMBA pulp does not form hydrogels, and can be reduced to a water content of 50\%, resulting in a potential reduction in transportation costs of the pulp. The chemicals used in the process are very common and not particularly expensive. Moreover, it is possible through well-established processes in the paper industry to reclaim these chemicals. The process directly affords acetylated fibres, which results in easy dispersion by low energy magnetic stirring. The prepared dispersions were seen to contain nanofibres, with no large aggregates present, as illustrated by both SEM and TEM as well as through preparation of highly translucent CNF films. In an attempt to improve the film forming properties of the CNF films the alkali residue was reintroduced into the CNF films, which lead not only to an overall higher mass yield, but also to flatter films, which illustrated the potential of the residue as a novel cementing agent for the CNF films. Additionally, the inclusion of the alkali residue resulted in a significant reduction in the UV transmittance.

\section{ACKNOWLEDGEMENTS}

The author would like to acknowledge the FP7 - People - 2011, ITN Marie Curie International Training Network (ITN), COST Action FP1003 and COST Action FP1105 for financial support. Lars Schulte is acknowledged for his assistance with the microscopy analyses, Richard Andersson for carrying out the transmission electron microscopy and Sebastien Raynaud for assisting with measuring the optical properties of the composites with the Hazemeter. This paper is in memoriam of Professor Iñaki Mondragon Egaña, whose dedication is a great source of inspiration for the first author.

\section{REFERENCES}

Abdul Khalil HPS, Davoudpour Y, Islam MN, Mustapha A, Sudesh K, Dungani R, Jawaid M (2014) Production and modification of nanofibrillated cellulose using various mechanical processes: a review. Carbohydr Polym 99:649-65. doi: 10.1016/j.carbpol.2013.08.069

Aulin C, Gällstedt M, Lindström T (2010) Oxygen and oil barrier properties of microfibrillated cellulose films and coatings. Cellulose 17:559-574. doi: 10.1007/s10570-009-9393-y 
Azizi Samir MAS, Alloin F, Dufresne A (2005) Review of recent research into cellulosic whiskers, their properties and their application in nanocomposite field. Biomacromolecules 6:612-26. doi: 10.1021/bm0493685

Baker E, Keisler JM (2011) Cellulosic biofuels: Expert views on prospects for advancement. Energy 36:595605. doi: 10.1016/j.energy.2010.09.058

Belbekhouche S, Bras J, Siqueira G, Chappey C, Lebrun L, Khelifi B, Marais S, Dufresne A (2011) Water sorption behavior and gas barrier properties of cellulose whiskers and microfibrils films. Carbohydr Polym 83:1740-1748. doi: 10.1016/j.carbpol.2010.10.036

Bismarck A, Aranberri-Askargorta I, Springer J, Mohanty AK, Misra M, Hinrichsen G, Czapla S (2001) Surface characterization of natural fibers; surface properties and the water up-take behavior of modified sisal and coir fibers. Green Chem 3:100-107. doi: 10.1039/b100365h

Chang C, Zhang L (2011) Cellulose-based hydrogels: Present status and application prospects. Carbohydr Polym 84:40-53. doi: 10.1016/j.carbpol.2010.12.023

Czaja WK, Young DJ, Kawecki M, Brown RM (2007) The future prospects of microbial cellulose in biomedical applications. Biomacromolecules 8:1-12. doi: 10.1021/bm060620d

de Morais Teixeira E, Corrêa AC, Manzoli A, de Lima Leite F, de Oliveira CR, Mattoso LHC (2010) Cellulose nanofibers from white and naturally colored cotton fibers. Cellulose 17:595-606. doi: 10.1007/s10570010-9403-0

Eichhorn SJ, Dufresne A, Aranguren M, Marcovich NE, Capadona JR, Rowan SJ, Weder C, Thielemans W, Roman M, Renneckar S, Gindl W, Veigel S, Keckes J, Yano H, Abe K, Nogi M, Nakagaito A. N, Mangalam A, Simonsen J, Benight A. S, Bismarck A, Berglund L A., Peijs T (2009) Review: current international research into cellulose nanofibres and nanocomposites. J Mater Sci 45:1-33. doi: 10.1007/s10853-009-3874-0.

Fischer F, Rigacci A., Pirard R, Berthon-Fabry S, Achard P (2006) Cellulose-based aerogels. Polymer 47:7636-7645. doi: 10.1016/j.polymer.2006.09.004

Henriksson M, Berglund L A., Isaksson P, Lindström T, Nishino T (2008) Cellulose nanopaper structures of high toughness. Biomacromolecules 9:1579-1585. doi: 10.1021/bm800038n

Henriksson M, Henriksson G, Berglund LA, Lindström T (2007) An environmentally friendly method for enzyme-assisted preparation of microfibrillated cellulose (MFC) nanofibers. Eur Polym J 43:3434-3441. doi: 10.1016/j.eurpolymj.2007.05.038

Herrick FW, Casebier RL, Hamilton KJ, Sandberg KR (1983) Microfibrillated Cellulose: Morphology and Accessibility. J Appl Polym Sci Appl Polym Symp 37:797-813.

Kim D, Nishiyama Y, Kuga S (2002) Surface acetylation of bacterial cellulose. Cellulose 361-368.

Lavoine N, Desloges I, Dufresne A, Bras J (2012) Microfibrillated cellulose - its barrier properties and applications in cellulosic materials: a review. Carbohydr Polym 90:735-64. doi: 10.1016/j.carbpol.2012.05.026

Lin N, Dufresne A (2014) Nanocellulose in biomedicine: Current status and future prospect. Eur Polym J 59:302-325. doi: 10.1016/j.eurpolymj.2014.07.025

Minelli M, Baschetti MG, Doghieri F, Ankerfors M, Lindström T, Siró I, Plackett D (2010) Investigation of mass transport properties of microfibrillated cellulose (MFC) films. J Memb Sci 358:67-75. doi: 10.1016/j.memsci.2010.04.030

Mondragon G, Fernandes S, Retegi A, Peña C, Algar I, Eceiza A, Arbelaiz A (2014) A common strategy to extracting cellulose nanoentities from different plants. Ind Crops Prod 55:140-148. doi: 10.1016/j.indcrop.2014.02.014

Moon RJ, Martini A, Nairn J, Simonsen J, Youngblood J (2011) Cellulose nanomaterials review: structure, properties and nanocomposites. Chem Soc Rev 40:3941-94. doi: 10.1039/c0cs00108b

Mwaikambo LY, Ansell MP (1999) The effect of chemical treatment on the properties of hemp, sisal, jute and kapok for composite reinforcement. Die Angew Makromol Chemie 272:108-116. doi: 10.1002/(SICI)1522-9505(19991201)272:1<108::AID-APMC108>3.0.CO;2-9 
Qing Y, Sabo R, Zhu JY, Agarwal U, Cai Z, Wu Y (2013) A comparative study of cellulose nanofibrils disintegrated via multiple processing approaches. Carbohydr Polym 97:226-34. doi: 10.1016/j.carbpol.2013.04.086

Rodionova G, Lenes M, Eriksen Ø, Gregersen Ø (2010) Surface chemical modification of microfibrillated cellulose: improvement of barrier properties for packaging applications. Cellulose 18:127-134. doi: 10.1007/s10570-010-9474-y

Saheb DN, Jog JP (1999) Natural fiber polymer composites: A review. Adv Polym Technol 18:351-363. doi: 10.1002/(SICI)1098-2329(199924)18:4<351::AID-ADV6>3.0.CO;2-X

Saito T, Nishiyama Y, Putaux JL, Vignon M, Isogai A (2006) Homogeneous suspensions of individualized microfibrils from TEMPO-catalyzed oxidation of native cellulose. Biomacromolecules 7:1687-1691. doi: 10.1021/bm060154s

Siqueira G, Bras J, Dufresne A (2010) New process of chemical grafting of cellulose nanoparticles with a long chain isocyanate. Langmuir 26:402-411. doi: 10.1021/la9028595

Siró I, Plackett D (2010) Microfibrillated cellulose and new nanocomposite materials: a review. Cellulose 17:459-494. doi: 10.1007/s10570-010-9405-y

Siró I, Plackett D, Hedenqvist M, Ankerfors M, Lindström T (2011) Highly transparent films from carboxymethylated microfibrillated cellulose: The effect of multiple homogenization steps on key properties. J Appl Polym Sci 119:2652-2660. doi: 10.1002/app.32831

Tejado A, Alam MN, Antal M, Yang H, van de Ven TGM (2012) Energy requirements for the disintegration of cellulose fibers into cellulose nanofibers. Cellulose 19:831-842. doi: 10.1007/s10570-012-9694-4

Trifol J, Plackett D, Sillard C, Hassager O, Daugaard AE, Bras J, Szabo P (2016a) A comparison of partially acetylated nanocellulose, nanocrystalline cellulose, and nanoclay as fillers for high-performance polylactide nanocomposites. J Appl Polym Sci 133:43257. doi: 10.1002/app.43257

Trifol J, Plackett D, Sillard C, Szabo P, Bras J, Daugaard AE (2016b) Hybrid poly(lactic acid)/nanocellulose/nanoclay composites with synergistically enhanced barrier properties and improved thermomechanical resistance. Polym Int. 65:988-995. doi: 10.1002/pi.5154

Wu C-N, Saito T, Yang Q, Fukuzumi H, Isogai A (2014) Increase in the Water Contact Angle of Composite Film Surfaces Caused by the Assembly of Hydrophilic Nanocellulose Fibrils and Nanoclay Platelets. ACS Appl Mater Interfaces 6:12707-12712. doi: 10.1021/am502701e 